\title{
AN EFFECTIVE AND ROBUST TECHNIQUE FOR THE BINARIZATION OF DEGRADED DOCUMENT IMAGES
}

\author{
Prashali Chaudhary $^{1}$, B.S. Saini ${ }^{2}$ \\ ${ }^{I}$ M.tech Scholar, Electronics and Communication Engineering, Dr. B. R. Ambedkar National Institute of Technology, \\ Punjab, India \\ ${ }^{2}$ Associate Professor, Electronics and Communication Engineering, Dr. B. R. Ambedkar National Institute of \\ Technology, Punjab, India
}

\begin{abstract}
Binarization methods play a central role in document image processing. It is usually performed in the preprocessing stage and is important for document image processing tasks such as optical character recognition (OCR). Segmentation of text from badly degraded document images is a challenging task because of the high inter/intra-variation between the document background and foreground text of different document images. So method for segmenting the foreground text from the background is presented here. In this method first of all an image having high contrast has been constructed. For this a rough estimation of background is to be made. Then a hybrid algorithm for thresholding has been used. It consists of both global and local thresholding methods. The Global thresholding step has been modified such that the output will not be a binarized image but an intermediate gray level image. It is helpful as most of the background gets eliminated. Local thresholding will be applied on the result given by global thresholding step. This method is simple, robust and effective. The proposed method works better than most of the existing local and global thresholding algorithms and is able to deal with degradations which occur due to strain, ink bleed through, low contrast, water marks, dust, smear and uneven illumination etc. This method has been tested on three public datasets that are used in recent document image binarization contest (DIBCO) 2009 and 2011 and handwritten-DIBCO 2011 and achieves the results which are significantly higher than or close to the best performing methods reported in three contests. Also to show the superior performance of the proposed method compared with other techniques, experiments have been performed on more challenging bickley diary dataset.
\end{abstract}

Keywords: Document image analysis, Thresholding, target pixels.

\section{INTRODUCTION}

Historical documents are of great importance to us. Many efforts or programs at national and international level aim to preserve a large number of historical documents so that a more efficient information access can be done. These historical documents are to be converted into digital form for convenient and easy storage and therefore majority of the projects are based on this. Severe degradations of historical documents quality is caused due to aging, chemical procedure of paper fabrication and storage conditions. So for preserving the information contained in these documents we follow a technique called binarization.

Binarization of document images is a challenging and old problem for Document Image Analysis and Retrieval (DIAR). The aim is to segment the pixels of the image in just two classes i.e. foreground and background. It is important for document image processing tasks such as optical character recognition (OCR) and usually performed in the preprocessing stage.

Several binarization techniques were proposed and developed in literature. They can be categorized as global, local and hybrid methods. Global binarization or we can say global thresholding methods compute an optimal threshold for the entire image. These techniques work well for simple cases and require less computation. But these techniques fail if the background is complex or non uniform in color or poorly illuminated. In case of local binarization different thresholds for different target pixels are set depending on the information extracted from the local neighborhood. These techniques are more efficient in case of bleed through degradation or poorly illuminated document and are also sensitive to background noise. A combination of global and local binarization will give us a hybrid binarization technique.

Many techniques for thresholding [1]-[4] have been reported for document image binarization. Global thresholding is usually not a suitable approach for the degraded document binarization as many degraded documents do not have a clear bimodal pattern. Brink [5], Kittler [6], Otsu [7] and Gatos [8] are some of the Global thresholding methods. Adaptive thresholding [9]-[15], is often a better approach to deal with different variations within degraded document images as it estimates a local threshold for each document image pixel. For example, the early window-based adaptive thresholding techniques [13], [14] estimate the local threshold by using the mean and the standard variation of image pixels within a local neighborhood window. These window based techniques have a drawback that the thresholding performance depends heavily on the window 
size and hence the character stroke width. Many other approaches have also been reported, including background subtraction [16], [17], texture analysis [19], recursive method [20], [21], Markov Random Field [22]-[25], matched wavelet [26], self-learning [27], Laplacian energy [28] user assistance [29], [30] and combination of binarization techniques [31], [32]. These methods combine different types of image information and domain knowledge and are often complex.

The objective of this work is to propose a thresholding algorithm which gives better binarization results which are free from degradations such as smear, uneven illumination and bleed through. The paper is organized as follows. In Section II the proposed method is presented. Measurements of binary image quality are introduced in Section III. Experimental results are shown in Section IV. Finally a conclusion is drawn in Section V.

\section{THE PROPOSED METHOD}

The proposed method requires the image to be preprocessed first. After that an estimated background is subtracted from the gray level filtered image and global binarization is applied. On the resultant image from the above step local thresholding is applied. Fig 1 shows the methodology followed to achieve the required binarization.

\subsection{Pre-Processing}

A preprocessing stage is necessary for the elimination of noisy areas from the source image. Gray scale image will be used i.e. image will be having 0 to 255 levels where 0 corresponds to black and 255 corresponds to white. We have used a Gaussian filter for the elimination of noise. Although wiener filter can also be used as it has proven to be efficient. So a gray scale source image will be transformed into a filtered gray scale image. Also the edges are to be preserved so this step is important.

\subsection{Background Subtraction}

As the document image is having degradations we can make a rough estimation of background. The image is first dilated and then eroded morphologically. By doing so we are left with a rough estimation of the background in which no foreground text appears. Now this estimated background is subtracted from the filtered gray scale image. The resultant image will be an image having a high contrast. Now any thresholding algorithm will give us good results as most of the unwanted regions will be eliminated and an optimal value of threshold can be calculated. Figure 2 shows the resultant image after background subtraction.

\subsection{Global Threshold}

Now a global threshold is chosen for the entire image. Since Otsu thresholding method [7] has greater superiority and reasonable complexity compared to other global thresholding methods, we chose this method. Generally applying a global threshold gives us a binary image but we have modified this. The outcome of this step will be an image having gray scale values for the pixels that belong to the foreground and value 255 i.e. white for the background pixels. As a result we have an image which is an intermediate gray scale image. This means:

$$
T(x, y)=\left\{\begin{array}{c}
I(x, y) \text { if } I(x, y) \leq t_{g} \\
255 \text { if } I(x, y)>t_{g}
\end{array}\right.
$$

Where, $T(x, y)$ is the value assigned to the pixel after the global binarization step. $\mathrm{I}(\mathrm{x}, \mathrm{y})$ is the gray value of the pixels and $t_{\mathrm{g}}$ is the global threshold that is computed for the entire image. Otsu thresholding method preserves a maximum number of foreground pixels and also it generates less background pixels misclassification at the first stage.

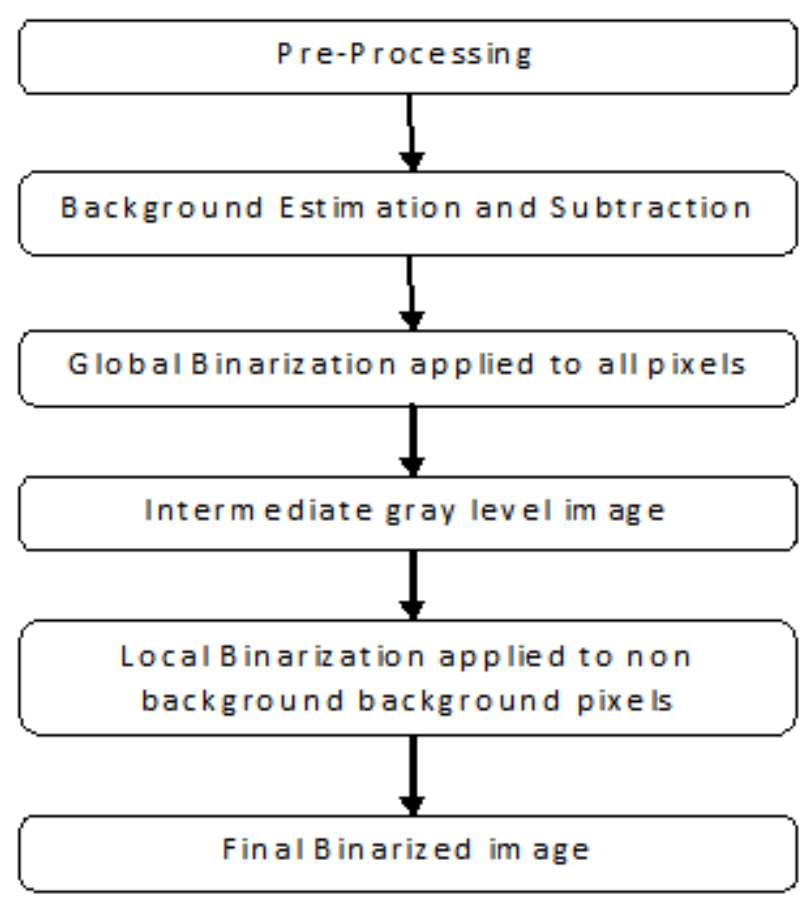

Fig -1: Methodology

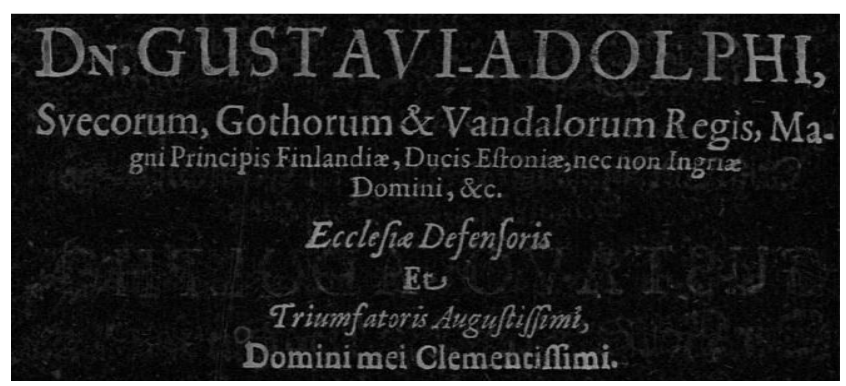

Fig -2: High contrast image after background subtraction

\subsection{Local Threshold}

Although the outcome of the previous step contains most of the background pixels separated from the foreground but some parts of the foreground still need to be clearly distinguished. Since some of the background pixels were earlier detected as foreground, these pixels are to be assigned to background class. So there is a requirement of Local thtesholding step. Local thresholding means 
calculating a threshold for a particular region or a window. This method is applied to all of the non background pixels. Savoula's method [13] has been used here. This technique provides us with a local threshold within the dynamic range of R. The equation for Savoula's binarization formula is:

$$
T(x, y)=m(x, y) \cdot\left[1+k \cdot\left(\frac{s(x, y)}{R}-1\right)\right]
$$

Where, $\mathrm{m}(\mathrm{x}, \mathrm{y})$ is the local mean, $\mathrm{s}(\mathrm{x}, \mathrm{y})$ is the local standard deviation and $\mathrm{k}$ is a constant. We have chosen the value of $\mathrm{R}=128, \mathrm{k}=0.3$ and window size $=16$. There parameters were chosen by experiments and they seem to give optimal results for Savoula's binarization in our case. This method gives better results for segmentation of background or foreground pixels.

\subsection{Post Processing}

The binarization results can further be improved by some post processing. For this firstly, the foreground pixels that do not connect with other foreground pixels are filtered out. Secondly we will check if the pixels on symmetric sides of any of the pixels belong to same class i.e. foreground or not. If both pixels belong to same class but center pixel belong to another class then that pixel will be assigned as foreground pixel. Also single pixel artifacts will be removed.

\section{EVALUATION MEASURES}

For comparing the results of different binarization approaches some measurements of the image quality are to be computed. There exist different methods for the measurement of image quality. These methods can be grouped as analytical, empirical goodness and the empirical discrepancy groups. Analytical methods easily obtain the properties of binarization algorithm by analysis. In these methods it is not possible to analyze the other properties as no formal model exists. The empirical goodness methods are based on judging the quality of binarized image with certain quality measures generated according to human intuition. It has been stated by Fung [33] that the experiments show that the discrepancy methods are more effective than the empirical goodness methods. Whereas, the methods which use difference between a binarized image and ground truth image to compared and evaluate the performance of binarization algorithms are called empirical discrepancy methods. In this paper we use four of these measures to compare the proposed method to other methods.

\subsection{F- Measure}

Basically F-measure is the harmonic mean of precision and recall. This measure combines the precision and recall as follows

$$
F-\text { measure }=\frac{2 \times \text { Precision } \times \text { Recall }}{\text { Precision }+ \text { Recall }}
$$

Where

$$
\text { Recall }=\frac{T P}{T P+F N}, \quad \text { Precision }=\frac{T P}{T P+F P}
$$

TP i.e. True Positives is the number of pixels which are classified as foreground in the binarized image and which are actually the foreground pixels in the ground truth image. FN i.e. False Negatives is the number of pixels which are classified as foreground in the binarized image but are background pixels in the ground truth image. FN i.e. False Negatives is the number of pixels classified as background in the binarized image which are actually the foreground pixels in the ground truth image.

A high value of F-measure indicates that the binarized image and the ground truth image are approximately equivalent. Also it implies that precision and recall are having high values.

\subsection{Peak Signal to Noise Ratio}

PSNR is commonly used to measure the closeness of one image to another. A higher value of PSNR indicates the higher similarity of two images. $\mathrm{C}$ is considered as the difference between foreground and the background.

$$
P S N R=10 \cdot \log f\left(\frac{C^{2}}{M S E}\right)
$$

Where,

$$
M S E=\frac{\sum_{x=1}^{M} \sum_{y=1}^{N}\left(I(x, y)-I^{\prime}(x, y)\right)^{2}}{M . N}
$$

$\mathrm{I}(\mathrm{x}, \mathrm{y})$ is the binarized image pixel value compared with $I^{\prime}(x, y)$ which is the ground truth image pixel value at the same pixel coordinates $(\mathrm{x}, \mathrm{y})$.

\subsection{Negative Rate Metric}

NRM is based on pixel wise mismatches between the ground truth and the predicted image. It combines the false negative rate $\mathrm{NR}_{\mathrm{FN}}$ and false positive rate $\mathrm{NR}_{\mathrm{FP}}$.

$$
N R M=\frac{N R_{F N}+N R_{F P}}{2}
$$

Where,

$N R_{F N}=\frac{N_{F N}}{N_{F N}+N_{T P}}, N R_{F P}=\frac{N_{F P}}{N_{F P}+N_{F N}}$

\subsection{Distance Reciprocal Distortion Metric}

DRD is used to measure the distortion in binary document images [13]. This measure correlates with human visual perception properly and measures the distortion for all the $\mathrm{S}$ flipped pixels according to following formula

$$
D R D=\frac{\sum_{k=1}^{S} D R D_{k}}{N U B N}
$$

Here, $\mathrm{DRD}_{\mathrm{k}}$ is the distortion of the k-th flipped pixel. It is calculated using $5 \times 5$ normalized weight matrix $\mathrm{W}_{\mathrm{Nm}}$. DRD equals to the weighted sum of the pixels in the $5 \times 5$ block of the Ground Truth $\boldsymbol{G} \boldsymbol{T}$ that differ from the centered k-th flipped pixel at $(\mathrm{x}, \mathrm{y})$ in the Binarization result image $\boldsymbol{B}$. 


$$
D R D_{k}=\sum_{i=-2}^{2} \sum_{j=-2}^{2}\left|G T_{k}(i, j)-B_{k}(x, y)\right| W_{N m}(i, j)
$$

NUBN is the number of the non-uniform $8 \times 8$ blocks in the GT image. Here non- uniform means not all black or white pixels. A low value of DRD is preferable.

\subsection{Misclassification Penalty Metric}

A prediction against the Ground Truth (GT) image is evaluated on an object-by-object basis. Misclassification pixels are penalized by their distance from the ground truth object's border providing us with a value of MPM.

$$
M P M=\frac{M P_{F N}+M P_{F P}}{2}
$$

Where,

$$
M P_{F N}=\frac{\sum_{i=1}^{N_{F N}} d_{F N}^{i}}{D}
$$

\section{EXPERIMENTS AND DISCUSSION}

To demonstrate the effectiveness and robustness of our proposed method a few experiments are designed. The proposed method is tested and compared with state-of-theart-methods over on three well known competition datasets. These datasets are DIBCO 2009 dataset [1], H-DIBCO 2010 dataset [2] and DIBCO 2011 dataset [3]. Further the proposed technique is evaluated over a very challenging Bickley diary dataset [34]. Some examples from the DIBCO 2011 dataset are shown in Fig. 3(a) and 4(a) with their binarized results 3(b) and 4(b) respectively.

The binarization performance is evaluated by using Fmeasure, Peak Signal to Noise Ratio (PSNR), Negative Rate Metric (NRM), Misclassification Penalty Metric (MPM) and Distance Reciprocal Distortion Metric (DRD). No all the metrics are applied on every image due to lack of ground truth image in some datasets.

The dataset contains images which represent degradations such as variable background intensity, low contrast, shadows, bleed-through, smear, smudge etc. which appear frequently. We quantitatively compare our proposed method with other state-of-the-art techniques on DIBCO 2011 dataset. These methods include Otsu's method (OTSU) [7], Sauvola's method (SAUV) [13], Niblack's method (NIBL) [14], Bernsen's method (BERN) [9], Gatos et al.'s method (GATO) [8], LMM [17] and BE [16] methods by Su, Lu and Tan [4]. The evaluation results of different methods including the proposed binarization method on DIBCO images database are presented in table 1, 2 and 3 for DIBCO 2009, H-DIBCO 2010 and DIBCO 2011 respectively. From table 1 and 2 we can analyze that our method performs best in terms of F-measure, PSNR, NRM and MPM. This means that our proposed method produces a higher overall precision and preserves the text strokes better. The values of DRD and MPM should be low and F- measure and PSNR should have high values. Table 3 shows that this method performs best in terms of DRD and MPM which means that this method provides best visual quality and maintains good text stroke contours. F-measure and PSNR values are very close to the highest scores.

Table -1: Evaluation results of the dataset of dibco 2009

\begin{tabular}{|l|l|l|l|l|}
\hline Methods & $\begin{array}{l}\text { FM } \\
(\%)\end{array}$ & PSNR & $\begin{array}{l}\text { NRM } \\
\left(\times 10^{-2}\right)\end{array}$ & $\begin{array}{l}\text { MPM } \\
\left(\times 10^{-3}\right)\end{array}$ \\
\hline OTSU [7] & 78.72 & 15.34 & 5.77 & 13.3 \\
\hline SAUV [13] & 85.41 & 16.39 & 6.94 & 3.2 \\
\hline NIBL [14] & 55.82 & 9.89 & 16.4 & 61.5 \\
\hline BERN [9] & 52.48 & 8.89 & 14.29 & 113.8 \\
\hline GATO [8] & 85.25 & 16.5 & 10 & 0.7 \\
\hline LMM [17] & 91.06 & 18.5 & 7 & 0.3 \\
\hline BE [16] & 91.24 & 18.6 & 4.31 & 0.55 \\
\hline SU [4] & 93.5 & 19.65 & 3.74 & 0.43 \\
\hline PROPOSED & 93.82 & 19.67 & 3.27 & 0.24 \\
\hline
\end{tabular}

Table -2: Evaluation results of the dataset of h- dibco 2010

\begin{tabular}{|l|l|l|l|l|}
\hline Methods & $\begin{array}{l}\text { FM } \\
(\%)\end{array}$ & PSNR & $\begin{array}{l}\text { NRM } \\
\left(\times 10^{-2}\right)\end{array}$ & $\begin{array}{l}\text { MPM } \\
\left(\times 10^{-3}\right)\end{array}$ \\
\hline OTSU [7] & 85.27 & 17.51 & 9.77 & 1.35 \\
\hline SAUV [13] & 75.3 & 15.96 & 16.31 & 1.96 \\
\hline NIBL [14] & 74.1 & 15.73 & 19.06 & 1.06 \\
\hline BERN [9] & 41.3 & 8.57 & 21.18 & 115.98 \\
\hline GATO [8] & 71.99 & 15.12 & 21.89 & 0.41 \\
\hline LMM [17] & 85.49 & 17.83 & 11.46 & 0.37 \\
\hline BE [16] & 86.41 & 18.14 & 9.06 & 1.11 \\
\hline SU [4] & 92.03 & 20.12 & 6.14 & 0.25 \\
\hline PROPOSED & 92.89 & 21.00 & 5.68 & 0.17 \\
\hline
\end{tabular}

Table -3: Evaluation results of the dataset of dibco 2011

\begin{tabular}{|l|l|l|l|l|}
\hline Methods & $\begin{array}{l}\text { FM } \\
(\%)\end{array}$ & PSNR & DRD & $\begin{array}{l}\text { MPM } \\
\left(\times 10^{-3}\right)\end{array}$ \\
\hline OTSU [7] & 82.22 & 15.77 & 8.72 & 15.64 \\
\hline SAUV [13] & 82.54 & 15.78 & 8.09 & 9.20 \\
\hline NIBL [14] & 68.52 & 12.76 & 28.31 & 26.38 \\
\hline BERN [9] & 47.28 & 7.92 & 82.28 & 136.54 \\
\hline GATO [8] & 82.11 & 16.04 & 5.42 & 7.13 \\
\hline LMM [17] & 85.56 & 16.75 & 6.02 & 6.42 \\
\hline BE [16] & 81.67 & 15.59 & 11.24 & 11.40 \\
\hline LELO [35] & 80.86 & 16.13 & 104.48 & 64.43 \\
\hline HOWE [27] & 88.74 & 17.84 & 5.37 & 8.64 \\
\hline SU [4] & 87.80 & 17.56 & 4.84 & 5.17 \\
\hline PROPOSED & 87.83 & 17.79 & 3.96 & 3.29 \\
\hline
\end{tabular}

To show the robustness and superior performance of our method we evaluated our method on Bickley Diary Dataset. The images from Bickley diary dataset are taken from a photocopy of a diary that is written about 100 years ago. These are more challenging than the previous DIBCO datasets. The evaluation results are shown in Table- 4 . 
Table -4: Evaluation Results of Bickley Diary Dataset

\begin{tabular}{|l|l|l|l|l|}
\hline Methods & $\begin{array}{l}\text { FM } \\
(\%)\end{array}$ & PSNR & $\begin{array}{l}\text { NRM } \\
\left(\times 10^{-2}\right)\end{array}$ & $\begin{array}{l}\text { MPM } \\
\left(\times 10^{-3}\right)\end{array}$ \\
\hline OTSU [7] & 50.42 & 7.58 & 21.41 & 196.98 \\
\hline SAUV [13] & 64.60 & 11.62 & 23.26 & 28.97 \\
\hline NIBL [14] & 67.71 & 9.79 & 9.52 & 105.17 \\
\hline BERN [9] & 52.97 & 7.71 & 18.86 & 193.35 \\
\hline GATO [8] & 69.13 & 11.44 & 21.89 & 36.57 \\
\hline LMM [17] & 66.44 & 10.76 & 17.50 & 72.08 \\
\hline BE [16] & 34.65 & 3.54 & 40.78 & 370.15 \\
\hline SU [4] & 78.54 & 13.15 & 12.92 & 16.71 \\
\hline PROPOSED & 78.78 & 13.50 & 12.07 & 12.10 \\
\hline
\end{tabular}

\section{CONCLUSIONS}

Development of thresholding algorithms for historical documents is crucial and necessary. The results of traditional approaches based either on the calculation of a global or local threshold are highly dependent on the type of the document image degradation and the quality of the binarization is significantly affected in some cases (for example, the global thresholding is not suitable for stained paper).

Firstly this method makes use of background subtraction which provides us with an image which is high in contrast. This helps to suppress the background variation and avoid the over-normalization of the document images with less variation. The method used for background estimation is better than other methods like polynomial smoothing. Secondly, Otsu's method is used for getting most of the background eliminated. Majority of the background pixels are separated from the foreground. Thirdly, the background pixels which were estimated as foreground are eliminated in local thresholding step. This method is tolerant to different types of degradations such as uneven illumination and document smear. The proposed technique is simple and robust. Also it works for different types of degraded document images. This method has been tested on DIBCO 2011 dataset. Experiments show that the proposed method outperforms most of the document binarization methods in terms of F-measure, PSNR, NRM, MPM and DRD.

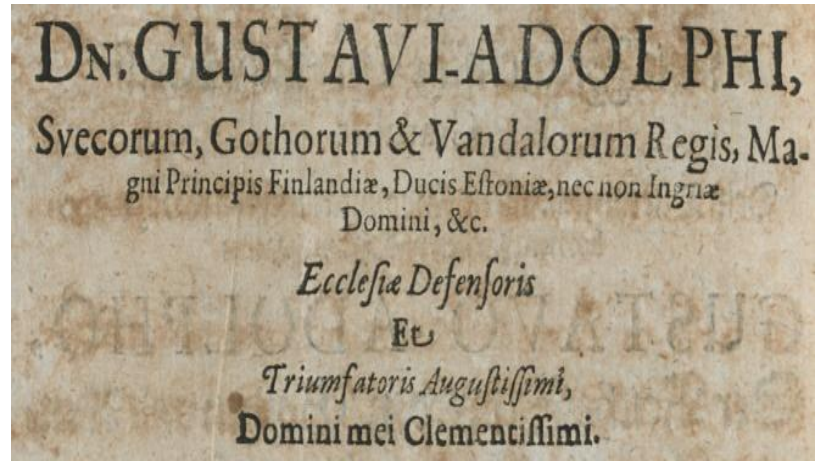

Fig -3(a): Original Image

\section{DN.GUST AVI-ADOLPHI,} $\underset{\text { Sve }}{\text { gni Principis Funland } x, \text { Ducis Eftonta, nec non Ingrix }}$, MaDomni , \&c.

Ecclefia Defenforis

Et

Triumfatoris Auguftifjemi, Domini mei Clementifimi.

Fig -3(b): Binarized Result

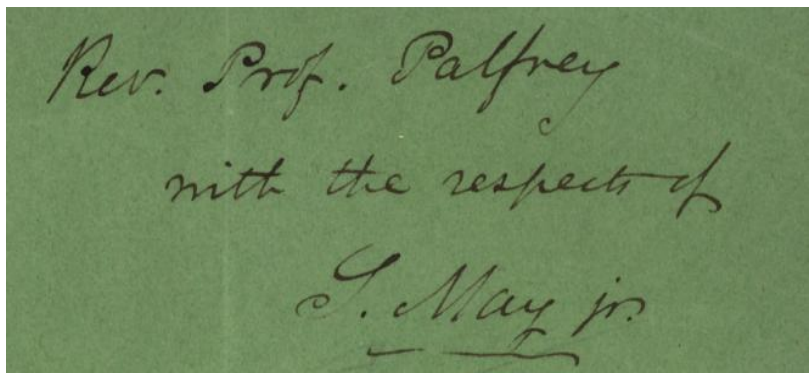

Fig -4(a): Original Image

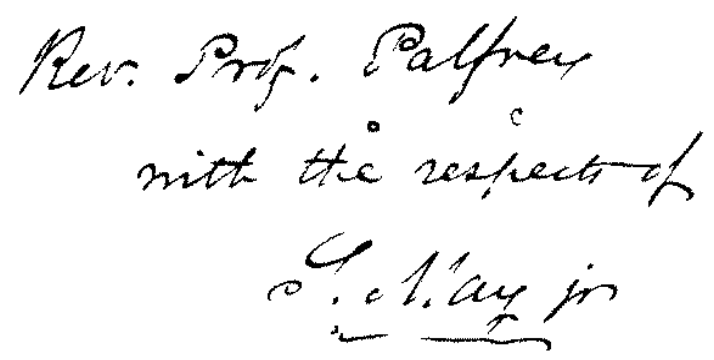

Fig -4(b): Binarized Result

\section{REFERENCES}

[1]. B. Gatos, K. Ntirogiannis, and I. Pratikakis, "ICDAR 2009 document image binarization contest (DIBCO 2009)," in Proc. Int. Conf. Document Anal. Recognit., Jul. 2009, pp. 1375-1382.

[2]. I. Pratikakis, B. Gatos, and K. Ntirogiannis, "H-DIBCO 2010 handwritten document image binarization competition," in Proc. Int. Conf. Frontiers Handwrit. Recognit., Nov.2010, pp. 727-732.

[3]. I. Pratikakis, B. Gatos, and K. Ntirogiannis, "ICDAR 2011 document image binarization contest (DIBCO 2011)," in Proc. Int. Conf. Document Anal. Recognit., Sep. 2011, pp. 1506-1510.

[4]. B. Su, S. Lu and C.L. Tan, "Robust Document Image Binarization Technique for Degraded Document Images", IEEE Trans. Image Process, vol. 22, no 4, pp. 1408-1417, April 2013.

[5]. A. Brink, "Thresholding of digital images using twodimensional entropies," Pattern Recognit., vol. 25, no. 8, pp. 803-808,1992.

[6]. J. Kittler and J. Illingworth, "On threshold selection using clustering criteria," IEEE Trans. Syst., Man, Cybern., vol. 15 , no. 5, pp. 652-655, Sep.-Oct. 1985.

[7]. N. Otsu, "A threshold selection method from gray level histogram," IEEE Trans. Syst., Man, Cybern., vol. 19, no. 1, pp. 62-66, Jan. 1979. 
[8]. N. Papamarkos and B. Gatos, "A new approach for multithreshold selection," Comput. Vis. Graph. Image Process., vol. 56, no. 5, pp. 357-370, 1994.

[9]. J. Bernsen, "Dynamic thresholding of gray-level images," in Proc. Int.Conf. Pattern Recognit., Oct. 1986, pp. 1251-1255.

[10]. L. Eikvil, T. Taxt, and K. Moen, “A fast adaptive method for binarization of document images," in Proc. Int. Conf. Document Anal. Recognit., Sep. 1991, pp. 435-443. [11]. I.K. Kim, D.W. Jung, and R.H. Park, "Document image binarization based on topographic analysis using a water flow model," Pattern Recognit., vol. 35, no. 1, pp. 265-277, 2002.

[12]. J. Parker, C. Jennings, and A. Salkauskas, "Thresholding using an illumination model," in Proc. Int. Conf. Doc. Anal. Recognit., Oct. 1993, pp. 270-273.

[13]. J. Sauvola and M. Pietikainen, "Adaptive document image binarization," Pattern Recognit., vol. 33, no. 2, pp. 225-236, 2000.

[14]. W. Niblack, An Introduction to Digital Image Processing. Englewood Cliffs, NJ: Prentice-Hall, 1986.

[15]. J.D. Yang, Y.S. Chen, and W.-H. Hsu, "Adaptive thresholding algorithm and its hardware implementation," Pattern Recognit. Lett., vol. 15, no. 2, pp. 141-150, 1994.

[16]. S. Lu, B. Su, and C. L. Tan, "Document image binarization using background estimation and stroke edges," Int. J. Document Anal. Recognit., vol. 13, no. 4, pp. 303314, Dec. 2010.

[17]. B. Su, S. Lu, and C. L. Tan, "Binarization of historical handwritten document images using local maximum and minimum filter," in Proc. Int. Workshop Document Anal. Syst., Jun. 2010, pp. 159-166.

[18]. B. Gatos, I. Pratikakis, and S. Perantonis, "Adaptive degraded document image binarization," Pattern Recognit., vol. 39, no. 3, pp. 317-327, 2006

[19]. Y. Liu and S. Srihari, "Document image binarization based on texture features," IEEE Trans. Pattern Anal. Mach. Intell., vol. 19, no. 5, pp. 540-544, May 1997.

[20]. M. Cheriet, J. N. Said, and C. Y. Suen, "A recursive thresholding technique for image segmentation," in Proc. IEEE Trans. Image Process., Jun. 1998, pp. 918-921.

[21]. S. Kumar, R. Gupta, N. Khanna, S. Chaudhury, and S. D. Joshi, "Iterative multimodel subimage binarization for handwritten character segmentation," IEEE Trans. Image Process., vol. 13, no. 9, pp. 1223-1230, Sep. 2004.

[22]. S. Nicolas, J. Dardenne, T. Paquet, and L. Heutte, "Document image segmentation using a 2D conditional random field model," in Proc. Int. Conf. Doc. Anal. Recognit., Sep. 2007, pp. 407-411.

[23]. C. Wolf and D. Doermann, "Binarization of low quality text using a markov random field model," in Proc. Int. Conf. Pattern Recognit., 2002, pp. 160-163.

[24]. T. Lelore and F. Bouchara, "Document image binarisation using Markov field model," in Proc. Int. Conf. Doc. Anal. Recognit., Jul. 2009, pp. 551-555.

[25]. J. G. Kuk, N. I. Cho, and K. M. Lee, "Map-MRF approach for binarization of degraded document image," in Proc. Int. Conf. Image Process., 2008, pp. 2612-2615.

[26]. S. Kumar, R. Gupta, N. Khanna, S. Chaudhury, and S. D. Joshi, "Text extraction and document image segmentation using matched wavelets and MRF model," IEEE Trans. Image Process., vol. 16, no. 8, pp. 2117-2128, Aug. 2007. [27]. B. Su, S. Lu, and C. L. Tan, "A self-training learning document binarization framework," in Proc. Int. Conf. Pattern Recognit., Aug. 2010, pp. 3187-3190.

[28]. N. Howe, "A Laplacian energy for document binarization," in Proc. Int. Conf. Doc. Anal. Recognit., Sep. 2011, pp. 6-10.

[29]. F. Deng, Z. Wu, Z. Lu, and M. S. Brown, "Binarizatioinshop: A userassisted software suite for converting old documents to black-and-white," in Proc. Annu. Joint Conf. Digit. Libraries, 2010, pp. 255-258.

[30]. H. Yi, M. S. Brown, and X. Dong, "User-assisted inkbleed reduction," IEEE Trans. Image Process., vol. 19, no. 10, pp. 2646-2658, Oct. 2010.

[31]. E. Badekas and N. Papamarkos, "Optimal combination of document binarization techniques using a selforganizing map neural network.” Eng. Appl. Artif. Intell., vol. 20, no. 1, pp. 11-24, Feb. 2007.

[32]. B. Gatos, I. Pratikakis, and S. Perantonis, "Improved document image binarization by using a combination of multiple binarization techniques and adapted edge information," in Proc. Int. Conf. Pattern Recognit., Dec. 2008, pp. 1-4.

[33]. C. Fung and R. Chamchong, A Review of Evaluation of Optimal Binarization Technique for Character Segmentation in Historical Manuscripts, Third International Conference on Knowledge Discovery and Data Mining, pp. 236-240, 2010.

[34]. F. Deng, Z. Wu, Z. Lu, and M. S. Brown, "Binarizatioinshop: A userassisted software suite for converting old documents to black-and-white," in Proc. Annu. Joint Conf. Digit. Libraries, 2010, pp. 255-258.

[35]. T. Lelore and F. Bouchara, "Super-resolved binarization of text based $\mathrm{n}$ the fair algorithm," in Proc. Int. Conf. Document Anal. Recognit., Sep. 2011, pp. 839-843.

\section{BIOGRAPHIES}

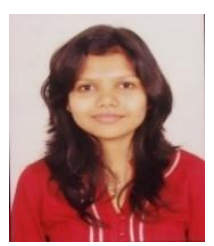

Prashali Chaudhary received her B.Tech degree in Electronics and communication Engineering from YMCAIE, Faridabad, Haryana in 2012 and currently pursuing M.Tech in Electronics and communication Engineering from Dr. B. R. Ambedkar National Institute of Technology Jalandhar, India.

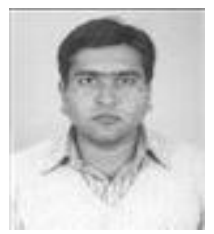

Barjinder Singh Saini received his B.Tech and M.Tech. degrees in Electronics \& Communication Engineering in 1994 and 1996 respectively. He then obtained his $\mathrm{PhD}$ degree in Engineering in 2009 from Dr. B. R. Ambedkar National Institute of Technology, Jalandhar. He is working as associate professor in Electronics \& Communication Engineering Department at NIT Jalandhar since 1997. 